\title{
O EIXO INTESTINO-CÉREBRO E O PAPEL DA SEROTONINA
}

\author{
Kleber Vedovato ${ }^{1}$ \\ Aline Rosa Trevizan ${ }^{2}$ \\ Caroline Nonis Zucoloto ${ }^{3}$ \\ Murillo Dorileo Leite Bernardi ${ }^{3}$ \\ Jacqueline Nelisis Zanoni ${ }^{4}$ \\ Juliana Vanessa Colombo Perles Martins ${ }^{4}$
}

VEDOVATO, K.; TREVIZAN, A. R.; ZUCOLOTO, C. N.; BERNARDI, M. D. L.; ZANONI, J. N.; MARTINS, J. V. C. P. O eixo intestinocérebro e o papel da serotonina. Arq. Ciênc. Saúde Unipar, Umuarama, v. 18 n. 1, p. 33-42, jan./abr. 2014

\begin{abstract}
RESUMO: Este trabalho teve por finalidade buscar informações na literatura que descrevam a interação entre o sistema nervoso central (SNC) e sistema nervoso entérico (SNE), e que demonstrem de que forma a serotonina pode atuar no "eixo intestino-cérebro". A serotonina (5-hidroxitriptamina, 5-HT) originalmente descoberta em 1937 por Vittorio Erspamer é uma indolamina, encontrada em células do trato gastrointestinal, plaquetas e no sistema nervoso central de mamíferos, sendo que cerca de $95 \%$ da 5-HT corpórea é produzida no intestino. De acordo com a literatura o trato gastrointestinal é inervado por cinco diferentes classes de neurônios: neurônios entéricos, aferentes vagal, aferentes da coluna vertebral, eferentes parassimpáticos e eferentes simpáticos. Os alvos primários da 5-HT que é secretado pelas células enteroendócrinas são as projeções intrínsecas constituída por neurônios aferentes primários da mucosa e plexo mientérico, e projeções extrínsecas, constituída por nervos que transmitem sensações gerais além de náuseas e desconforto para o sistema nervoso central. Após os dados compilados da literatura fica claro que o SNE desempenha papel singular, podendo agir de maneira independente, mas também mantém sinapses com o SNC, por meio do nervo vago. Dessa forma, ambos os sistemas podem influenciar a atividade um do outro mediante o "eixo intestino-cérebro". Adicionalmente a 5-HT apresenta-se como um importante neurotransmissor envolvido na interação entre o SNE o SNC, importante na regulação da sensação de motilidade e secreção intestinal e também atua na ativação e condução da informação ao SNC.
\end{abstract}

PALAVRAS-CHAVE: Sistema nervoso entérico; Sistema nervoso central; Intestino; Serotonina.

\section{THE BRAIN-INTESTINE AXIS AND THE ROLE OF SEROTONIN}

\begin{abstract}
This paper aims to seek literature information describing the interaction between the central and enteric nervous system, and to demonstrate how serotonin may be acting on the "brain-intestine axis." Serotonin, originally discovered in 1937 by Vittorio Erspamer, is an indoleamine found in cells in the gastrointestinal tract, platelets and in the central nervous system of mammals, with approximately $95 \%$ of body 5 -HT produced in the intestine. According to literature, the gastrointestinal tract is innervated by five different neuron classes: enteric neurons, vagal afferent, spinal afferent, parasympathetic efferent, and sympathetic efferent. The primary targets of serotonin secreted by entero-endocrine cells are the intrinsic projections composed by primary afferent neurons of the mucosa and myenteric plexus, and extrinsic projections, consisting of nerves that send general sensations as well as nausea and general discomfort to the central nervous system. After the data were compiled from the literature, it is clear that the enteric nervous system plays a unique role and can act independently, but it also keeps synapses in the central nervous system, through the vagus nerve. Therefore, both systems can influence the activity of each other through the "brain-intestine axis ". In addition, serotonin seems to be an important neurotransmitter involved in the interaction between the enteric nervous system and the central nervous system, important in regulation of motility sensation and intestinal secretion, as well as acting in the activation and conduction of information to the central nervous system.
\end{abstract}

KEYWORDS: Enteric nervous system; Central nervous system; Brain-Intestine axis; Serotonin.

\section{Introdução}

O sistema serotonérgico exerce um papel bem conhecido sobre a homeostase orgânica. Além de atuar sobre a modulação da motilidade gastrintestinal e função plaquetária, a serotonina (5-HT) participa efetivamente da regulação hidroeletrolítica, modulando a sede e o apetite, ingestão alimentar, balanço energético, regulação da emoção e processos do controle comportamental (MOHAMMAD-ZADEH; MOSES; GWALTNEY-BRANT, 2008).

A maior parte da serotonina corporal, cerca de 95\%, é produzida no trato gastrointestinal pelas células enteroendócrinas ou enterocromafins, no intestino, e pelos neurônios serotoninérgicos do sistema nervoso entérico (GERSHON,
2000; GERSHON, 2004; GERSHON; TACK, 2007). No sistema nervoso entérico, a 5-HT é um neurotransmissor excitatório considerado importante por ser responsável por estimular as contrações peristálticas e secretórias pós-prandial, além de ativar os nervos sensoriais extrínsecos (GERSHON; TACK, 2007).

Alterações no sistema serotoninérgico podem levar a disfunções do trato gastrointestinal. A síndrome do intestino irritável (SII), uma doença gastrointestinal funcional caracterizada por dor ou desconforto abdominal associados a alterações na característica das evacuações, está sendo tratada com medicamentos antidepressivos da classe de Inibidores Seletivos de Recaptação da Serotonina (CATAPANI, 2004; PASSOS; RAMOS, 2006).

DOI: https://doi.org/10.25110/arqsaude.v18i1.2014.5156

'Especializado em Anatomia e Histologia pela Universidade Estadual de Maringá, Campus Maringá. E-mail: klebervedovato@hotmail.com. ${ }^{2}$ Cursando Pós-graduação em Anatomia e Histologia pela Universidade Estadual de Maringá, Campus Maringá. E-mail: alininha_trevizan@hotmail.com. ${ }^{3}$ Acadêmicos do Curso de Graduação em Biomedicina da Universidade Estadual de Maringá - Campus Maringá. E-mail: carol_nonis@hotmail.com; murillo_bernardi@hotmail.com.

${ }^{4}$ Docentes do Departamento de Ciências Morfológicas da Universidade Estadual de Maringá - Campus Maringá. E-mail: jnzanoni@uem.br; jjvcm77@ gmail.com.

Endereço para correspondência: Avenida Colombo, 5790, Bloco H79, Maringá, PR, Brazil. CEP: 87020-900. Fax: +554432614340. Telefone: +554430114691. Email: jjvcm77@gmail.com. 
Vários estudos recentes indicam uma alteração na sinalização e disponibilidade de serotonina em pacientes com síndrome do intestino irritável, e o desenvolvimento de medicamentos durante os últimos anos tem focado as drogas que interagem com mecanismos serotoninérgicos do intestino (OHMAN; SIMRÉN, 2007).

Pesquisas vem demonstrando um aumento da liberação e/ou redução na recaptação de serotonina pós-prandiais envolvidos na diarréia predominante em pacientes com SII (HOUGHTON et al., 2003; ATKINSON et al., 2006), enquanto liberação prejudicada parece ser uma característica de pacientes com constipação predominante (DUNLOP et al., 2005; ATKINSON et al., 2006).

As alterações da regulação das conexões do SNC com o intestino têm sido muito estudadas em pacientes com SII e, provavelmente, centros neurais superiores modulam as atividades motora e sensorial gastrintestinal, e vice-versa (PASSOS; RAMOS, 2006).

Passos; Ramos (2006) admite uma integração das atividades motoras, sensoriais, autonômicas do trato gastrointestinal, interagindo continuamente com o SNC. Para ele, informações exteriores ou cognitivas, podem estimular centros nervosos que interferem na função gastrintestinal, podendo influenciar a secreção, a motilidade e as sensações digestivas. Alguns estudos sugerem que o SNC processa anormalmente informação na SII (ATKINSON et al., 2006). Se essas observações forem confirmadas, a associação entre as alterações de personalidade e os transtornos funcionais provavelmente terão como base uma disfunção central.

Diante do exposto fica claro que o SNE apesar de agir de maneira independente, mantém sinapses com o SNC. Assim sendo, este trabalho teve como objetivo buscar informações na literatura que descrevam a interação entre o SNC e SNE, e que demonstrem de que forma a serotonina pode atuar nesse eixo.

\section{Desenvolvimento}

\section{Serotonina}

A 5-HT foi originalmente descoberta em 1937 por Vittorio Erspamer, em células enterocromafins na mucosa gastrointestinal de vertebrados, com ação de contração muscular, por ter sido identificada primeiramente no trato gastrointestinal foi chamada enteramina. Page e colaboradores no ano de 1948, subsequentemente, isolaram e caracterizaram em seus estudos de hipertensão uma substância endógena que causava vasoconstrição, que foi então chamada serotonina. Os pesquisadores observaram um fator do soro (latim: serum) que afetava o tônus vascular e mostrou-se idêntica ao indol anteriormente isolado por Erspamer. A ação neurotransmissora da serotonina foi descoberta posteriormente por Betty Twarog no músculo retrator do mexilhão em 1952 e depois em 1953 no SNC de mamíferos (JACOBS; AZMITIA, 1992; SANDERS-BUSH; MAYER, 1996).

Produto da hidroxilação e descarboxilação do aminoácido L-triptofano, a serotonina é uma indolamina, encontrada nas células enterocromafins do trato gastrointestinal, sendo o restante encontrado nas plaquetas e no SNC de mamíferos. Diversos estudos têm mostrado sua participação na modulação da motilidade gastrointestinal, tônus vascular pe- riférico, tônus vascular cerebral, além de exercer importante função plaquetária e na fisiopatologia de diversas desordens de humor, vômito, enxaquecas, SII e hipertensão sistêmica e pulmonar (MOHAMMAD-ZADEH; MOSES; GWALTNEY-BRANT, 2008).

No interior dos neurônios, a serotonina é produzida a partir do aminoácido triptofano, primeiramente convertido a 5-hidroxitriptofano pela enzima triptofano hidroxilase, que é então descarboxilado a 5-HT pela enzima 5-hidroxitriptofano descarboxilase. Após sua síntese, é estocada em grânulos secretórios através de um transportador vesicular e liberada para a fenda sináptica por exocitose dos neurônios serotoninérgicos. Sua metabolização ocorre pela ação da enzima monoamina oxidase e pela enzima aldeído desidrogenase originando seu metabólito principal, o ácido 5-hidroxiindolacético (5-HIAA) (SANDERS-BUSH; MAYER, 1996; LAM; HEISLER, 2007).

No SNC, neurônios contendo 5-HT são encontrados no mesencéfalo, ponte e bulbo, e estão essencialmente confinados às zonas mediana e paramediana nos chamados núcleos da rafe. Cada núcleo da rafe envia projeções para várias regiões do encéfalo. Aqueles mais caudais, no bulbo, inervam a medula espinal, onde modulam sinais sensoriais relativos à dor. Aqueles mais rostrais, na ponte e no mesencéfalo, inervam a maior parte do encéfalo, de forma difusa. No SNC a serotonina está envolvida no controle do apetite, sono, humor, alucinações, comportamento estereotipado e percepção da dor entre outros (MARTINS; SILVA; GLORIAM, 2010; MELLO, 2010).

Ainda no SNC a 5-HT é um neurotransmissor responsável pela transmissão eletroquímica sináptica através da ligação com receptores específicos, chamados serotoninérgicos, abundantes no sistema nervoso central, sendo os principais subtipos de receptores no sistema nervoso central: $5-\mathrm{HT}_{1 \mathrm{~A}}, 5-\mathrm{HT}_{1 \mathrm{~B}}, 5-\mathrm{HT}_{1 \mathrm{D}}, 5-\mathrm{HT}_{2}, 5-\mathrm{HT}_{3}$ (GOODMAN; GILMAN, 1996; MELO, 2010).

Apesar da importância e diversidade de mecanismos serotoninérgicos no SNC, o encéfalo não é o principal produtor de serotonina, na verdade ele contém muito pouca serotonina em termos relativos (ERSPAMER, 1966). A maior parte da serotonina presente no organismo humano, cerca de $95 \%$, é produzida no trato gastrointestinal. Desse percentual, aproximadamente $90 \%$ é sintetizado em células enteroendócrinas, um subtipo de células enterocromafins, e $10 \%$ nos neurônios entéricos. Essa 5-HT, presente no trato gastrintestinal age como um neurotransmissor das funções sensório-motoras gastrintestinais (KIM; CAMILLERI, 2000; COATES et al., 2006; GERSHON; TACK, 2007).

As células enteroendócrinas contêm uma taxa de biossíntese de serotonina limitada pela quantidade da enzima triptofano hidroxilase-1 (TPH-1) (COTE et al., 2003; WALTHER et al., 2003), enquanto que nos neurônios serotoninérgicos, tanto do SNC quanto entérico, contém outra variante da enzima, a TPH-2, que é um produto de gene diferente (COTE et al., 2003). As células enteroendócrinas produzem e secretam muito mais serotonina em comparação aos neurônios serotoninérgicos centrais ou periféricos, de forma que a serotonina secretada pelas células enteroendócrinas podem ser liberadas para o lúmen gastrointestinal (GRONSTARD et al., 1985; WINGREN et al., 1988) e o sangue (MORRISSEY; WALKER; LOVENBERG, 1977; TAMIR et al., 1985; 
CHEN et al., 2001).

Dessa forma, após a 5-HT ser sintetizada pelas células enteroendócrinas da mucosa intestinal (GERSHON, 2004) parte, é usada localmente como um neurotransmissor do SNE (GERSHON; TACK, 2007; CHEN et al., 2001) enquanto outra parte da 5 -HT se difunde do intestino para a circulação sanguínea, onde a maior parte é rapidamente eliminada pelo fígado e pulmões (THOMAS; VANE, 1967; ANDERSON; STEVENSON; COHEN, 1987), restando apenas a serotonina que é captada pelas plaquetas, única fonte de serotonina sérica (ERSPAMER, 1966; MORRISSEY; WALKER; LOVENBERG, 1977).

Sendo assim como as plaquetas sanguíneas não possuem a enzima TPH, portanto, não podem produzir serotonina por conta própria, elas captam ativamente 5-HT a partir do plasma sanguíneo utilizando uma bomba molecular, o transportador de serotonina (SERT) (LESURTEL et al., 2008). A 5-HT plaquetária está relacionada com o aumento da permeabilidade vascular; vasodilatação; contração da musculatura lisa e agente mitogênico potente que estimula a mitose de células de músculo liso, dos megacariócitos e dos fibroblastos (JULIUS et al., 1989; YANG et al., 1996; FANBURG; LEE, 1997; JUNQUEIRA; CARNEIRO, 2008)

No SNE os alvos primários da serotonina sintetizada pelas células enteroendócrinas, são as projeções primárias de neurônios da mucosa, que incluem os nervos extrínsecos (BLACKSHAW; GRUNDY, 1993; GRUNDY; BLACKSHAW; HILLSLEY, 1994; HILLSLEY; GRUNDY, 1998; HILLSLEY; KIRKUP; GRUNDY, 1998; SUGIUAR; BIELEFELDT; GEBHART, 2004) que transmitem sensações de náuseas e o desconforto para o $\mathrm{SNC}$, e as projeções de mucosa formados por neurônios intrínsecos primários aferentes (intrinsic primary afferent neurons - IPANs), os IPANs submucosos iniciam reflexos peristálticos e secretórios (KIRCHGESSNER; TAMIR; GERSHON, 1992; KIRCHGESSNER; LIU; GERSHON, 1996; PAN; GERSHON, 2000; SIDHU; COOKE, 1995; COOKE; SIDHU; WANG, 1997; KIM et al., 2001) enquanto que o IPANs mioentéricos medeiam as neurotransmissões excitatórias rápida e lenta e estão envolvidas na regulação da motidade gastrintestinal (GERSHON, 2004; GERSHON; TACK, 2007).

\section{Inervação Intrínseca do Sistema Digestório: Sistema Ner- voso Entérico e Atuação da Serotonina}

O sistema nervoso intrínseco é formado pelos circuitos neurais que constituem o SNE, responsável pelo comportamento motor do trato gastrointestinal e também por conduzir informações sobre o estado do trato gastrointestinal para o SNC por meio dos neurônios extrínsecos primários aferentes (COSTA; BROOKES, 1994; FURNESS; KUNZE; CLERC, 1999).

Durante muito tempo o SNE foi definido como a porção pós-ganglionar da divisão parassimpática do sistema nervoso autônomo. Atualmente é conhecido como um componente do sistema nervoso autônomo com função independente do SNC (FURNESS, 2006).

O SNE é constituído por uma rede de gânglios inseridos na parede do trato gastrointestinal e interconectados por fibras nervosas. Estende-se da faringe ao esfíncter anal, onde os plexos ganglionados mioentérico e submucoso exercem um importante papel no controle da motilidade, proliferação celular, transporte de íons pela mucosa e liberação de hormônios gastrintestinais. O SNE com seus plexos mioentérico e submucoso difere do sistema nervoso autônomo simpático e parassimpático em estrutura e função (FURNESS; COSTA, 1980; FURNESS, 2006).

Embora os plexos mioentérico e submucoso sejam espacialmente separados, a conexão entre os dois sugere que eles compreendem uma unidade integradora (FURNESS; COSTA, 1980; COSTA; FURNESS, 1987). O SNE possui essa autonomia por que seus gânglios contêm neurônios aferentes sensoriais, interneurônios e neurônios motores, que são os componentes neurais necessários para um circuito reflexo completo (BROOKES, 2001; FURNESS et al., 2003).

Os neurônios sensoriais, atualmente denominados como neurônios intrínsecos primários aferentes, traduzem e codificam informações sobre o ambiente químico e estado físico do tecido que eles inervam. Nos plexos mioentérico e submucoso, esses neurônios se conectam a outros neurônios intrínsecos primários aferentes, interneurônios e neurônios motores (DOGIEL, 1899; GERSHON; KIRCHGESSNER, 1991; COSTA; BROOKES; HENNIG, 2000). Os interneurônios são identificados em todas as camadas do trato gastrintestinal, sendo que sua constituição neuroquímica varia muito, dependendo do órgão em questão (FURNESS et al., 1995).

Os neurônios motores, por sua vez são divididos em neurônios excitatórios, neurônios inibitórios da musculatura lisa do intestino, e neurônios secretomotores/vasodilatadores. Os dois primeiros encontram-se no plexo mioentérico e estão envolvidos no controle da motilidade, já os neurônios secretomotores/vasodilatadores são encontrados no plexo submucoso e são responsáveis pela inervação da mucosa e pela regulação da secreção, absorção e do fluxo sanguíneo (FURNESS, 2000; FURNESS, 2006).

Os principais neuromediadores encontrados nos neurônios excitatórios são a acetilcolina e as taquicininas (substância P). Os neurônios inibitórios possuem vários neuromediadores, como NO (óxido nítrico), VIP (peptídeo intestinal vasoativo) e ATP (adenosina trifosfato) (FURNESS et al., 1995).

Diversas outras substâncias, como a histamina, prostaglandinas, leucotrienos, interleucinas, proteases e a serotonia, são capazes de modular a atividade neuronal entérica. A secreção de 5-HT no intestino, se da em resposta a uma ampla variedade de estímulos incluindo acidificação, instilação de glicose hipertônica ou solução de sacarose, aumento da pressão intraluminal, obstrução mecânica do intestino, isquemia intestinal e inclusive por ação do sistema nervoso central através da estimulação vagal (LI et al., 2000; SCHWERTZ; BRADESI; MAYER, 2003; MEARIN et al., 2004).

Os corpos de neurônios serotoninérgicos estão presentes apenas no plexo mioentérico, e possuí traços característicos do tipo Dogiel I, e fibras nervosas serotoninérgicas estão distribuídas em ambos os plexos mioentérico e submucoso (COSTA et al., 1982; LEGAY; SAFFREY; BURNSTOCK, 1984; NADA; TOYOHARA, 1987; WARDELL; BORNSTEIN; FURNESS, 1994; BARBIERS et al., 1995). As fibras serotoninérgicas estão densamente localizados ao redor de vasos sanguinos na tela submucosa observadas em 
todos os segmentos do trato gastrointestinal. A possibilidade de uma origem extrínseca destas fibras tem sido apontada (NADA; TOYOHARA, 1987), além da possibilidade, que não pode ser descartada, de que exista fibras perivasculares noradrenérgicas contendo 5-HT (FUJIMIYA et al., 1997).

As células enteroendócrinas liberam 5-HT em respostas a diferentes estímulos, como distensão mecânica pelo alimento, estímulos mecânicos das vilosidades da mucosa, produtos bacteriano como toxina da cólera, drogas citostáticas, e consequentemente, deflagram reflexos entéricos capazes de alterar a secreção intestinal ou a contração muscular (RAYBOULD, 1999; HOLZER et al., 2001; HARA et al., 2004).

A 5-HT liberada a partir de células enteroendócrinas pode estimular tanto neurônios aferentes primários (sensorial) intrínsecos e extrínsecos, através de pelo menos três diferentes receptores serotoninérgicos, 5-HT3, 5-HT4 e 5-HT1P (BERTRAND; FURNESS; BORNSTEIN, 1999; JIN; FOXX-ORENSTEIN; GRIDER, 1999; KIRCHGESSNER; TAMIR; GERSHON, 1992; PAN; GERSHON, 2000; ZHU et al., 2001). No SNE a estimulação de neurônios intrínsecos primários aferentes do plexo submucoso predominantemente através de receptores 5 HT1P e 5-HT4 (COOKE, 1997; PAN; GERSHON, 2000) e neurônios intrínsecos primários aferentes do plexo mioentérico preferencialmente via 5-HT3 (BERTRAND; FURNESS; BORNSTEIN, 1999; HOLZER et al., 2001; HARA et al., 2004).

A 5-HT ativa diretamente tanto a contração das células musculares lisas, como atua indiretamente na contração muscular por meio da estimulação de neurônios colinérgicos no plexo mioentérico (BULBRING; GERSHON, 1967; COSTA; FURNESS, 1979). No plexo submucoso a serotonina aumenta a secreção de água e eletrólitos na mucosa por estimulação indireta de ambos os neurônios colinérgicos e não-colinérgicos no plexo submucoso (CASSUTO et al., 1982; COOK; REDDIX, 1994; KUWAHARA; KUWAHARA; KADOWAKI, 1994).

Entretanto a função de modulação do trato gastrointestinal pela serotonina é estabelecida não só pela sua ação em nervos intrínsecos, mas também em nervos extrínsecos que, possuindo os receptores serotoninérgicos, sinalizam aos neurônios aferentes modificações presentes na mucosa do trato digestório (MEARIN et al., 2004; SCHWERTZ; BRADESI; MAYER, 2003).

\section{Inervações Extrínsecas do Sistema Digestório e Atuação da Serotonina}

É bem documentado que o SNE contém vias reflexas para a regulação da motilidade e da secreção, e que estes reflexos podem funcionar independentemente do sistema nervoso central. Entretanto a inervação extrínseca é considerada importante, pois transmite informações sobre o estado do trato gastrointestinal para o SNC sendo capaz de modular os reflexos intrínsecos entéricos (LARSSON et al., 2007).

Sendo assim, de forma geral, as funções nervosas do trato gastrointestinal são controladas pelo SNE (sistema nervoso intrínseco) e uma rede nervosa extrínseca composta pelos circuitos parassimpático e simpático que interagem com o sistema nervoso entérico (VAN KUYK et al., 2000).

$\mathrm{O}$ circuito parassimpático é abastecido pelos nervos vago e pélvico, dos quais longos axônios surgem dos corpos celulares da região sacral e cervical da medula espinal que chegam aos vários órgãos do trato gastrointestinal onde fazem sinapse com as células do SNE. O trajeto aferente projeta para o cérebro e medula espinal os estímulos sensoriais através da comunicação dos neurônios com receptores dispostos nos tecidos do intestino delgado (VAN KUYK et al., 2000).

Apesar da autonomia do SNE, neurônios eferentes vagais promovem uma inervação marcada por fibras profusas e generalizadas que chegam no plexo mioentérico do esôfago e do estômago, por outro lado a sua inervação no intestino delgado é menos extensa (BERTHOUD; LYNN; BLACKSHAW, 2001). Reflexos envolvendo neurônio aferentes e eferentes vagais, desempenham um papel proeminente no trato gastrointestinal superior, onde ajudam a coordenar as respostas motoras e secretoras da ingestão de alimentos. Embora a transmissão de impulsos nervosos eferentes através de receptores nicotínicos resultarem em excitação dos neurônios entéricos, a resposta final efetora depende das vias entéricas que estão sendo estimuladas no momento. Assim, os reflexos vagais motores podem resultar na contração muscular ou inibição da atividade muscular, como é o caso com o relaxamento receptivo do fundo gástrico (DESAI et al., 1991; TAKAHASHI; OWYANG, 1997), enquanto que influencia vagal sobre a secreção da região gastroduodenal são geralmente de natureza estimulante (WOOD; ALPERS; ANDREWS, 1999).

O circuito simpático difere pela presença dos gânglios celíaco, mesentérico superior e inferior que estão localizados no trajeto entre a medula espinal e trato gastrointestinal. As fibras eferentes pré-ganglionares se originam na medula espinal e terminam no gânglio pré-vertebral, as fibras pós-ganglionares continuam a transmissão do impulso inervando os plexos do SNE com inervações para músculos, mucosa e vasos sanguíneos que formam redes de varicosidades que permitem o controle independente da motilidade, secreção e fluxo sanguíneo. O caminho das fibras aferentes obedece ao mesmo raciocínio, transmitindo a informação sensorial para a medula espinal passando antes pelos gânglios (WEISBRODT, 1997; VAN KUYK et al., 2000).

O principal neurotransmissor liberado por neurônios eferentes pós-ganglionares simpáticos é a norepinefrina, enquanto a acetilcolina é o principal neurotransmissor secretado por neurônios eferentes parassimpáticos. Neuropeptídeos (por exemplo: NPY nos neurônios simpáticos, VIP nos neurônios parassimpáticos) também são co-liberados e estão envolvidos na neurotransmissão eferente. Estimulação das fibras eferentes simpáticas inibe a ativação dos neurônios entéricos, que posteriormente leva à diminuição da motilidade e secreção (LARSSON et al., 2007). Ativação das fibras parassimpáticas eferentes tem dado resultados contraditórios em animais, mostrando tanto excitação (GROAT; KRIER, 1976; GROAT; KRIER, 1978) quanto inibição (FASTH; HULTEN; NORDGREN, 1980).

A inervação extrínseca eferente que modula a atividade do SNE é relativamente escassa, com cerca de uma fibra eferente inervando 300 neurônios entéricos, enquanto que inervação extrínseca aferente é mais representativa, cerca de $90 \%$ do nervo vago consiste em fibras aferentes, e é responsável por transmitir informações a partir do intestino 
para o SNC (LARSSON et al., 2007).

Existem dois grupos de neurônios aferentes primários extrínsecos que inervam o intestino, os aferentes vagais provenientes do gânglio jugular (parte superior do trato digestório) e nodoso (parte inferior do trato digestório), e os aferentes espinais, que têm seus corpos celulares no gânglio da raiz dorsal. É importante ressaltar que $80-90 \%$ do axônio do nervo vago são fibras nervosas sensoriais e que, portanto, o nervo vago é, essencialmente, um nervo aferente. Os aferentes espinais suprem o trato gastrointestinal através dos nervos esplâncnicos e pélvicos, constituindo 10-30\% de todas as fibras nervosas (GRUNDY; SCRATCHERD, 1989; SENGUPTA; GEBHART, 1994). Nesta rota os axônios passam pelo gânglio pré-vertebral onde fazem, vias colaterais, sinapses com as células ganglionares simpáticas (GREEN; DOCKRAY, 1988; SZURSZEWSKI; MILLER, 1994).

Sendo assim, de forma geral, o trato gastrointestinal é inervado por cinco diferentes classes de neurônios: neurônios entéricos, aferentes vagal, aferentes da coluna vertebral, eferentes parassimpáticos e eferentes simpáticos.

No trato gastrointestinal, as terminações vagal e da coluna vertebral aferentes se iniciam na serosa, plexo mioentérico, camadas musculares, tela submucosa (principalmente arteríolas) e mucosa (GRUNDY; SCRATCHERD, 1989; SENGUPTA; GEBHART, 1994; CERVERO, 1994), onde captam alterações do ambiente químico no lúmen, espaço intersticial e vascular e às mudanças nas propriedades mecânicas da parede do intestino, como relaxamento, distensão ou contração (HOLZER et al., 2001).

Acreditava-se que sensações como dor decorrente das vísceras do trato gastrointestinal seriam mediadas exclusivamente por aferentes da coluna vertebral, enquanto que a única tarefa de aferentes vagais residia na regulação fisiológica da atividade digestiva no trato gastrointestinal superior (GRUNDY; SCRATCHERD, 1989; SENGUPTA; GEBHART, 1994; CERVERO, 1994). No entanto, pesquisas mais recentes demonstram uma consciência crescente de que aferentes vagal tenham uma contribuição relacionada com alterações na sensibilidade visceral à doença distintas (GEBHART, 2000; HOLZER et al., 2000). Neurônios vagais aferentes respondem a uma variedade de produtos químicos nocivos (GEBHART, 2000), e respostas a doenças que causam infecção e inflamação envolvem aferentes vagais que reagem à geração periférica da interleucina- $1 \beta$ e de outras citocinas pró-inflamatórias tendo como consequência febre, anorexia, sonolência, hiperalgesia, diminuição na atividade locomotora e na exploração social (DANTZER et al., 1998; MAIER et al., 1998).

Aferentes vagais sinalizam o insulto ao tronco medular e a informação é passada para mesencéfalo, núcleos talâmicos, hipotalâmico e sistema límbico (SCHULIGOI et al., 1998; MICHL et al., 2001). Assim, a sinalização aferente vagal de um insulto ácido agudo na mucosa gástrica leva a ativação de núcleos cerebrais subcorticais que estão envolvidos em reações autonômicas e neuroendócrinas, emocionais e comportamentais, a um estímulo nocivo (MICHL et al., 2001).

Além das citocinas pró-inflamatórias, pesquisas tem demonstrado que aferências extrínsecas vagais podem ser ativadas por colecistocinina, secretina, fator de liberação corticotropina, e somatostatina e serotonina que são substân- cias secretadas por células enteroendócrinas e imunes (BUÉNO et al., 1997; RAYBOULD, 1999; FURNESS; KUNZE; CLERC, 1999), que estão estrategicamente posicionados na mucosa do trato gastrointestinal para analisar o conteúdo luminal. A liberação dessas moléculas mensageiras podem estimular as fibras nervosas sensoriais que transmitem a informação emitida a partir do monitoramento da mucosa para o SNE e central (HOLZER et al., 2001).

Estudos demonstram que a liberação de 5-HT a partir de células enterocromafins intestinais atua sobre os receptores 5-HT3 em fibras aferentes vagais despolarizando os neurônios sensitivos dessa via, conduzindo os estímulos gerados por alterações da osmolaridade luminal e produtos da digestão de carboidrato até o SNC (hipotálamo) (ZHU et al., 2001; LI et al., 2003).

Estes mecanismos explicam alterações dos estados emocionais decorrentes de distúrbios intestinais, constipação ou diarreias, associados a SII. Atualmente a SII é vista como um distúrbio de desregulação do chamado eixo cérebro-intestino, envolvendo um funcionamento anormal nos sistemas nervosos entérico, autônomo e central, com alterações periféricas que provavelmente irão perturbar o próprio processamento central desses sinais emitidos por disturbios no trato gastrointestinal em alguns pacientes (LIN; KUNZE; STANIZ, 2004; LAUFFER, 2010).

$\mathrm{Na}$ SII o estímulo inicial parece se originar a partir de células enteroendócrinas que servem como transdutores de produtos químicos ou mecânicos para estímulos nocivos. (CAMILLERI et al., 2001). Degranulação dessas células libera serotonina, um neurotransmissor chave no SNE, que também irá estimular aferências extrínsecas (KYRLAGKITSIS; KARAMANOLIS; , 2002).

Em individuos saudáveis o SNC recebe continuamente informações de todo trato gastrointestinal, porém, em condições fisiológicas a maioria das informações não atinge o nível de consciência, portanto sem a nossa percepção, sendo processadas pelo hipotálamo. Por outro lado em indivíduos com Síndrome do Intestino Irritável ocorre percepção consciente do estimulo doloroso (KARAMANOLIS; KYRLAGKITSI, 2002).

O caminho para a transmissão do sinal por meio das aferências extrínsecas até o SNC é constituído por três vias neuronais. Na primeira, o corpo do neurônio sensitivo está no gânglio da raiz dorsal e termina na coluna dorsal de substância cinzenta da medula espinal. Na segunda via o corpo do neurônio encontra-se na coluna dorsal de substância cinzenta da medula espinal e projeta-se para a formação reticular do tronco cerebral e o tálamo, por meio do trato espinorreticular e espinotalâmico, respectivamente. Nessas áreas encefálicas, esses neurônios fazem sinapses com os centros autonômicos e com o neurônio que constituirá a terceira via de projeções para o sistema límbico (respostas emocionais) e para o córtex sensorial que leva à percepção consciente do estímulo doloroso (CAMILLERI; SASLOW; BHARUCHA, 1996; KARAMANOLIS; KYRLAGKITSIS, 2002).

As drogas mais indicadas para o alívio da dor abdominal são os antiespasmódicos, como os antimuscarínicos, trimebutina, brometo de pinavério, brometo de octilônio e mebeverina. Os bloqueadores do canal de cálcio não seletivos, como o diltiazen e a nifedipina, agem sobre o cólon, mas os seus efeitos sistêmicos restringem o uso na SII (BUÉNO 
et al., 1997).

Os antidepressivos tricíclicos (amitriptilina, imipramina, desipramina, doxepina) ou os inibidores da captação da serotonina (fluoxetina, sertralina, paroxetina), que apresentam como mecanismo de ação uma provável analgesia central, estão sendo utilizados no tratamento da SII (FORD et al., 2009).

Ambas as classes de drogas foram inicialmente utilizadas para tratar transtornos mentais co-existentes com a SII, como depressão e ansiedade, mas ensaios clínicos demonstraram que pacientes com a SII e sem transtorno depressivo foram beneficiados com doses baixas de terapia com antidepressivos tricíclicos (HALPER et al., 2005). Surpreendentemente, tanto antidepressivos tricíclicos quanto inibidores seletivos de recaptação de serotonina não interferem com as concentrações de serotonina no intestino, o que poderia contribuir para aumentar os sintomas de dor na SII. Em vez disso, eles parecem normalizar a motilidade do trato gastrointestinal reduzindo a dor visceral (KALL; LINDSTROM; MARTINEZ, 2007; FORD et al., 2009).

A redução da dor visceral provavelmente se deva a ação desses medicamentos nas vias eferentes autonômicas (serotoninérgicas, adrenérgicas) a partir dos centros do tronco cerebral, como a substância cinzenta periaquedutal, alterando a sensibilidade dos neurônios do corno dorsal da medula espinal, modulando a intensidade da percepção a dor durante a estimulação visceral (KARAMANOLIS; KYRLAGKITSIS, 2002).

\section{Interações entre o sistema nervoso entérico e central e o papel da serotonina}

A inervação paralela do intestino por neurônios aferentes intrínsecos e extrínsecos sugere que essas duas classes de neurônios sensoriais contribuem com dois papéis fundamentalmentais e diferentes na função gastrointestinal. Os neurônios sensoriais intrínsecos suprem o SNE com um tipo de informação que o SNE requer para seu controle, independente, da digestão, enquanto os aferentes extrínsecos conduzem ao sistema nervoso central informações que são relevantes para o controle de energia do corpo, de fluidos e eletrólitos (homeostase), da integridade dos tecidos e das sensações de desconforto e dor, promovendo uma interação, via sistema nervoso parassimpático, entre SNE e central (HOLZER et al., 2001).

Um importante neurotransmissor envolvido na interação entre o SNE e o SNC é a 5-HT, que atua na ativação e condução da informação ao SNC que responde com estímulo para a realização dos movimentos gastrointestinais (GERSHON, 2004; BERTRAND, 2006).

Os alvos primários da serotonina que é secretada pelas células enteroendócrinas são os neurônios aferentes primários das projeções da mucosa. Estes incluem as projeções intrínsecas constituida por neurônios aferentes primários da mucosa, e projeções extrínsecas, constituida por nervos que transmitem sensações gerais além de náuseas e desconforto para o SNC (GERSHON; JAN TACK, 2007).

A liberação de 5-HT no intestino provoca uma grande variedade de efeitos devido a ampla expressão e distribuição de vários subtipos de 5-HT, nesse órgão. Os mais estudados são os receptores 5-HT1P e 5-HT4 responsáveis por ativar a via intrínseca; e o receptor 5-HT3, presente nos neurônios sensitivos da via extrínseca (GERSHON, 1999).

A serotonina atua sobre os receptores 5-HT3 em fibras aferentes vagais, despolarizando os neurônios sensitivos dessa via desencadeando a ativação do nervo vago que conduzirá diversos estímulos, gerados no trato gastrointestinal, até o SNC (ZHU et al., 2001; LI et al., 2003). Estudo demonstrou que o antagonismo de receptores 5-HT3, em humanos, aumenta limiares sensoriais desencadeados por balão de distensão retal, evidências que podem fornecer um mecanismo para explicar os efeitos terapêuticos de antagonistas 5-HT3 na modulação da dor (GERSHON, 1999).

Dessa forma uma vez que os neurônios serotoninérgicos centrais são separados dos neurônios serotoninérgicos periféricos, plaquetas e células enteroendócrinas pela barreira hemato encefálica, que é impermeável à serotonina. (GERSHON; JAN TACK, 2007). Alterações na síntese e liberação de serotonina ocorrida no trato gastrointestinal são transmitidas ao SNC pelo nervo vago, que contém uma grande quantidade de fibras aferentes extrínsecas, provenientes do trato gastrointestinal (LARSSON et al., 2007).

\section{Conclusão}

Após os dados compilados da literatura fica claro que o SNE desempenha papel singular, podendo agir de maneira independente, mas também mantém sinapses com o SNC, dessa forma, ambos os sistemas podem influenciar a atividade um do outro através do "eixo intestino-cérebro", que é representado pela inervação intrínseca e extrínseca do trato gastrointestinal.

Adicionalmente a serotonina apresenta-se como um importante neurotransmissor envolvido na interação entre o SNE e o SNC, além de ser um dos neurotransmissores-chave dentro do trato gastrointestinal, ela afeta a sensação de motilidade e secreção intestinal e também atua na ativação e condução da informação ao sistema nervoso central.

\section{Referências}

ANDERSON, G. M.; STEVENSON, J. M.; COHEN, D. J. Steady-state model for plasma free and platelet serotonin in man. Life Sci. v. 41, p. 1777-1785, 1987.

ATKINSON, W. et al. Altered 5-hydroxytryptamine signaling in patients with constipation-and diarrheapredominant irritable bowel syndrome. Gastroenterology, v. 130, p. 34-43, 2006.

BARBIERS, M. et al. Projections of neurochemically specified neurons in the porcine colon. Histochemistry, v. 103, n. 2, p. 115-126, 1995.

BERTRAND, P. P. Real-time measurement of serotonin release and motility in guinea pig ileum. Journal of Physiology, London, v. 577, n. 2, p. 689-704, 2006.

BERTRAND, P. P.; FURNESS, J. B.; BORNSTEIN, J. C. 5-HT and ATP stimulate the mucosal terminals of some myenteric sensory neurons and cause increases in excitability in a population of sensory neurons. 
Neurogastroenterology, Freiburg, n. 112, 1999.

BERTHOUD, H. R.; LYNN, P. A.; BLACKSHAW, L. A. Vagal and spinal mechanosensors in the rat stomach and colon have multiple receptive fields. Am. J. Physiol. Regul. Integr. Comp. Physiol. v. 280, p. R1371-R1381, 2001.

BLACKSHAW, L. A.; GRUNDY, D. Effects of 5-hydroxytryptamine on discharge of vagal mucosal afferent fibres from the upper gastrointestinal tract of the ferret. $\mathbf{J}$. Auton. Nerv. Syst. v. 45, p. 41-50, 1993.

BROOKES, S. J. H. Classes of enteric nerve cells in the guinea-pig small intestine. Anat. Rec. v. 262, p. 58-70, 2001.

BUÉNO, L. et al. Mediators and pharmacology of visceral sensitivity: from basic to clinical investigations. Gastroenterology, v. 112, p. 1714-1743, 1997.

BÜLBRING, E.; GERSHON, M. D. 5-Hydroxytryptamine participation in the vagal inhibitory innervation of the stomach. J. Physiol. v. 192, p. 823-846, 1967.

CASSUTO, J. et al. 5-Hydroxytryptamine and cholera secretion: Physiological and pharmacological studies in cats and rats. Sand. J. Gastroenterol. v. 17, p. 695-703, 1982.

CAMILLERI, M.; S. B.; SASLOW, A. E.; BHARUCHA, A. Gastrointestinal sensation: Mechanisms and relation to functional gastrointestinal disorders. Gastroenterol. Clin. North. Am. v. 25, p. 247-258, 1996.

CAMILLERI, M. et al. Management of the irritable bowel syndrome. Gastroenterology, v. 120, p. 652-668, 2001.

CATAPANI, W. Conceitos atuais em síndrome do intestino irritável. Arq. Med. ABC, v. 29, n. 1, 2004.

CERVERO, F. Sensory innervation of the viscera: peripheral basis of visceral pain. Physiol. Rev. v. 74, p. 95138, 1994.

CHEN, J. J. et al. Maintenance of serotonin in the intestinal mucosa and ganglia of mice that lack the high-affinity serotonin transporter (SERT): Abnormal intestinal motility and the expression of cation transporters. J. Neurosci. v. 21, p. 6348-6361, 2001.

COATES, M. D. et al. Effects of serotonin transporter inhibition on gastrointestinal motility and colonic sensitivity in the mouse. Neurogastroenterol. Motil. v. 18, p. 464471, 2006.

COOK, H. J.; REDDIX, R. A. Neural regulation of intestinal electrolyte transport. In: JOHNSON, L. R. (Ed.). Physiology of the gastrointestinal. 1994.

COOKE, H. J.; SIDHU, M.; WANG, Y-Z. 5-HT activates neural reflexes regulating secretion in the guinea-pig colon. Neurogastroenterol. Motil. v. 9, p. 181-186, 1997.
COSTA, M.; BROOKES, S. J. H.; HENNIG, G. W. Anatomy and physiology of the enteric nervous system. Gut, v. 47, p. iv15-iv19, 2000.

COSTA, M.; BROOKES, S. J. H. The enteric nervous system. Am. J. Gastroenterol. v. 89, p. 125-137, 1994.

COSTA, M.; FURNESS, J. B. The sites of action of 5-hydroxytryptamine in nerve-muscle preparations from the guinea-pig small intestine and colon. Br. J. Pharmacol. v. 65 , p. $237-248,1979$.

. Neuronal peptides in the intestine. Britssh Medical Bulletin, v. 38, p. 247-252, 1987.

COSTA, M. et al. Neurons with 5-hydroxytryptaminelike immunoreactivity in the enteric nervous system: their visualization and reactions to drug treatment. Neuroscience, v. 7, n. 2, p. 351-363, 1982.

COTE, F. et al. Disruption of the nonneuronal tph1 gene demonstrates the importance of peripheral serotonin in cardiac function. Proc. Natl. Acad. Sci. v. 100, p. 1352513530, 2003.

DANTZER, R. et al. Cytokines and sickness behavior. Ann. N. Y. Acad. Sci. v. 840, p. 586-590, 1998.

DESAI, K. M. et al. Nitroxergic nerves mediate vagally induced relaxation in the isolated stomach of the guinea pig. Proc. Natl. Acad. Sci. v. 88, p. 11490-11494, 1991.

DOGIEL, A. S. Über den Bau der Ganglien in den Geflechten des Darmes und der Gallenblase dês Menschen und der Säugetiere. Arch. Anat. Physiol. p. 130-158, 1899.

DUNLOP, S. P. et al. Abnormalities of 5-hydroxytryptamine metabolism in irritable bowel syndrome. Clin.

Gastroenterol. Hepatol. v. 3, p. 349-357, 2005.

ERSPAMER, V. Occurrence of indolealkylamines in nature. In: ERSPAMER, V. (Ed.). Handbook of experimental pharmacology: 5-hydroxytryptamine and related indolealkylamines. New York: Springer-Verlag, 1966. p. 132-181.

FANBURG, B. L.; LEE, S. L. A new role for an old molecule: serotonin as a mitogen. Am. J. Physiol. v. 272, p. L795-806, 1997.

FASTH, S.; HULTEN, L.; NORDGREN, S. Evidence for a dual pelvic nerve influence on large bowel motility in the cat. J. Physiol. v. 298, p. 159-169, 1980.

FORD, A. C. et al. Efficacy of antidepressants and psychological therapies in irritable bowel syndrome: systematic review and meta-analysis. Gut, v. 58, p. 367378, 2009.

FUJIMIYA, M. et al. Distribution of serotoninimmunoreactive nerve cells and fibers in the rat gastrointestinal tract. Histochemistry And Cell Biology, v. 
107, n. 2, p.105-114, 1997.

FURNESS, J. B. The enteric nervious system. Austrália: Blackwell Publishing, 2006.

. Types of neurons in the enteric nervous system. $\mathbf{J}$. Auton. Nerv. Syste. v. 81, p. 87-96, 2000.

Types of nerves in the enteric nervous system. Neuroscience, v. 5, n. 1, p. 1-20, 1980.

FURNESS, J. B.; COSTA, M. The enteric nervous system. New York: Churchill Livingstone, 1987.

FURNESS, J. B. et al. Morphologies and projections of defined classes of neurons in the submucosa of the guineapig small intestine. Anat. Rec. v. 272A, p. 475-483, 2003.

FURNESS, J. B.; KUNZE, W. A. A.; CLERC, N. Nutrient tasting and signaling mechanisms in the gut. II. The intestine as a sensory organ: neural, endocrine, and immune responses. American Journal Physiology, v. 277, p. 922928, 1999.

FURNESS, J. B. et al. Plurichemical transmission and chemical coding of neurons in the digestive tract. Gastroenterology, v. 108, p. 554-563, 1995.

GEBHART, G. F. Pathobiology of visceral pain: molecular mechanisms and therapeutic implications: IV. Visceral afferent contributions to the pathobiology of visceral pain. Am. J. Physiol. v. 278, p. G834-G838, 2000.

GERSHON, M. D. Effect of tetrodotoxin on innervated smooth muscle preparations. Br. J. Pharmacol.

Chemother. v. 29, p. 259-279, 1967.

. 5-HT (serotonin) physiology and related drugs.

Curr. Opin. Gastroenterol. v. 16, p. 113-120, 2000. 1999.

O segundo cérebro. Rio de Janeiro: Campus,

. Review article: serotonin receptors and transporters - roles in normal and abnormal gastrointestinal motility. Alimentary Pharmacology and Therapeutics, Oxford, v. 20, n. 7, p. 3-14, 2004.

GERSHON, M. D.; KIRCHGESSNER, A. L. Identification, characterization and projections of intrinsic primary afferent neurones of the submucosal plexus: Activity- induced expression of c-fos immunoreactivity. Journal of the Autonomic Nervous System, v. 33, p. 185-187, 1991.

GERSHON, M. D.; TACK, J. The Serotonin Signaling System: From Basic Understanding To Drug Development for Functional GI Disorders. Gastroenterology, v. 132, p. 397-414, 2007.

GOODMAN, A.; GILMAN, P. As bases farmacológicas da terapêutica. 9. ed. Rio de Janeiro: McGraw-Hill, 1996.
GREEN, T.; DOCKRAY, G. J. Characterization of the peptidergic afferent innervation of the stomach in the rat, mouse, and guinea-pig. Neuroscience, v. 25, p. 181-193, 1988.

GRONSTARD, K. O. et al. The effects of vagal nerve stimulation on endoluminal release of serotonin and substance P into the feline small intestine. Scand. J. Gastroenterol. v. 20, p. 163-169, 1985.

GROAT, W. C.; KRIER, J. An electrophysiological study of the sacral parasympathetic pathway to the colon of the cat. J. Physiol. v. 260, p. 425-445, 1976.

The sacral parasympathetic reflex pathway regulating colonic motility and defaecation in the cat. $\mathbf{J}$. Physiol. v. 276, p. 481-500, 1978.

GRUNDY, D.; BLACKSHAW, L. A.; HILLSLEY, K. Role of 5-hydroxytryptamine in gastrointestinal chemosensitivity. Dig. Dis. Sci. v. 39, n. 12, p. S44-S47, 1994.

GRUNDY, D.; SCRATCHERD, T. Sensory afferents from the gastrointestinal tract. In: SCHULTZ, S. G. Handbook of Physiology: The Gastrointestinal System, Motility and Circulation. Bethesda: American Physiological Society, 1989. p. 593-620.

HALPERT, A. et al. Clinical response to tricyclic antidepressants in functional bowel disorders is not related to dosage. Am. J. Gastroenterol. v. 100, p. 664-671, 2005.

HARA, J. R. et al. Enteroendocrine cells and 5-HT availability are altered in mucosa of guinea pigs with TNBS ileitis. Am. J. Physiol. Gastrointest. Liver Physiol. v. 287, p. G998-G1007, 2004.

HILLSLEY, K.; GRUNDY, D. Sensitivity to 5-hydroxytryptamine in different afferent subpopulations within mesenteric nerves supplying the rat jejunum. J. Physiol. v. 509, p. 717-727, 1998.

HILLSLEY, K.; KIRKUP, A. J.; GRUNDY, D. Direct and indirect actions of 5-hydroxytryptamine on the discharge of mesenteric afferent fibers innervating the rat jejunum. J. Physiol. v. 506, p. 551-561, 1998.

HOLZER, P. et al. Essential role of vagal afferents in the central signalling of a gastric mucosal acid insult. In: KRAMMER, H. J.; SINGER, M. V.

Neurogastroenterology. Dordrecht: From the Basics to the Clincs. Kluwer Academic Publishing, 2000. p. 697-707.

HOLZER, P. et al. Inflammation and gut hypersensitivity: peripheral mechanisms. Gastroenterol. v. 14, p. 32-45, 2001.

HOUGHTON, L. A. et al. Increased platelet depleted plasma 5-hydroxytryptamine concentration following meal ingestion in symptomatic female subjects with diarrhoea predominant irritable bowel syndrome. Gut, v. 52, p. 663- 
670, 2003.

JACOBS, B. L.; AZMITIA, E. C. Structure and function of the brain serotonin system. Physiological Reviews, v. 72, p. 165-229, 1992.

JIN, J. G.; FOXX-ORENSTEIN, A. E.; GRIDER, J. R. Propulsion in guinea pig colon induced by 5-hydroxytryptamine (HT) via 5-HT4 and 5-HT3 receptors. J. Pharmacol. Exp. Ther. v. 288, p. 93-97, 1999.

JULIUS, D. et al. Ectopic expression of the serotonin 1c receptor and the triggering of malignant transformation. Science, v. 244, p. 1057-1062, 1989.

JUNQUEIRA, L. C. U.; CARNEIRO, J. Histologia básica. 11. ed. Rio de Janeiro: Guanabara Koogan, 2008.

KALL, E.; LINDSTROM, E.; MARTINEZ, V. The serotonin reuptake inhibitor citalopram does not affect colonic sensitivity or compliance in rats. Eur. J.

Pharmacol. v. 570, p. 203-211, 2007.

KYRLAGKITSIS, I. KARAMANOLIS, D. G. Pathophysiology of Irritable bowel syndrome: The role of brain-gut axis and serotoninergic receptors. Annals of gastroenterology, v. 15, n. 3, p. 248-252, 2002.

KIM, M. et al. D-glucose releases 5-hydroxytryptamine from human BON cells as a model of enterochromaffin cells. Gastroenterology, v. 121, p. 1400-1406, 2001.

KIM, D. Y.; CAMILLERI, M. Serotonin: a mediator of the brain-gut connection. Am. J. Gastroenterol. v. 95, p. 2698 2709, 2000.

KIRCHGESSNER, A. L.; LIU, M. T.; GERSHON, M. D. In situ identification and visualization of neurons that mediate enteric and enteropancreatic reflexes. J. Comp. Neurol. v. 371, p. 270-286, 1996.

KIRCHGESSNER, A. L.; TAMIR, H.; GERSHON, M. D. Identification and stimulation by serotonin of intrinsic sensory neurons of the submucosal plexus of the guinea pig gut: activity-induced expression of Fos immunoreactivity. J. Neurosci. v. 12, p. 235-249, 1992.

KUWAHARA, A.; KUWAHARA, Y.; KADOWAKI, M. Analysis of FK1052, a new potent 5HT3 and 5HT4 receptor dual antagonist of guinea-pig distal colon. Biomed. Res. v. 3, p. 205-210, 1994.

LAM, D. D.; HEISLER, L. K. Serotonin and energy balance: molecular mechanisms and implications for type 2 diabetes.

Expert Rev. Mol. Med. v. 9, n. 5, p. 1-24, 2007.

LARSSON, M. H. et al. Elevated motility-related transmucosal potential difference in the upper small intestine in the irritable bowel syndrome.

Neurogastroenterol. Motil. v. 19, n. 10, p. 812-820, 2007.

LAUFFER, A. Tradução e validação para o português do Brasil do questionário "Patient Assessment Of Upper Gastrointestinal Disorders-Quality Of Life (PAGI-QOL)" em pacientes dispépticos funcionais. 2010. Dissertação (Mestrado em Medicina: Ciências em Gastroenterologia) - Universidade Federal do Rio Grande do Sul, Faculdade de Medicina, Porto Alegre, 2010.

LEGAY, C.; SAFFREY, M. J.; BURNSTOCK, G.

Coexistence of immunoreactive substance $\mathrm{P}$ and serotonin in neurons of the gut. Brain Res. v. 302, p. 379-382, 1984.

LESURTEL, M. et al. Role of serotonin in the hepatogastroIntestinal tract: an old molecule for new perspectives. Cell Mol. Life Sci. v. 65, p. 940-952, 2008.

LI, Y. et al. Serotonin released from intestinal enterochromaffin cells mediates luminal non-cholecystokinin-stimulated pancreatic secretion in rats. Gastroenterology, v. 118, p. 11971207, 2000.

LI, Y. et al. Hypothalamic regulation of pancreatic secretion is mediated by central cholinergic pathways in the rat. $\mathbf{J}$.

Physiol. v. 552, n. 2, p. 571-587, 2003.

LIN, J.; KUNZE, W.; STANIZ, A. Inflammation of mouse jejunum induces long term excitation in DRG neurons projecting to the viscera. Gastroenterology, v. 126A, p. 896, 2004.

MAIER, S. F. et al. The role of the vagus nerve in cytokineto-brain communication. Ann. N. Y. Acad. Sci. v. 840, p. 289-300, 1998.

MARTINS, A. C. C. L.; SILVA, T. M.; GLORIAM, B. A. Determinação simultânea de precursores de serotonina triptofano e 5-hidroxitriptofano - em café. Química Nova, v. 33, n. 2, p. 316-320, 2010.

MEARIN, F. et al. Spittling irritable bowel syndrome: from original Rome to Rome II criteria. Am. J. Gastroenterol. v. 99 , p. 122-130, 2004.

MELO, S. R. Neuroanatomia: pintar para aprender. São Paulo: Roca, 2010.

MICHL, T. et al. Vagal afferent signaling of a gastric mucosal acid insult to medullary, pontine, thalamic, hypothalamic and limbic, but not cortical, nuclei of the rat brain. Pain, v. 92, p. 19-27, 2001.

MOHAMMAD-ZADEH, L. F.; MOSES, L.; GWALTNEYBRANT, S. M. Serotonin: a review. J. Vet. Pharmacol. Ther. v. 31, n. 3, p. 187-99, 2008.

MORRISSEY, J. J.; WALKER, M. N.; LOVENBERG, W. The absence of tryptophan hydroxylase activity in blood platelets. Proc. Soc. Exp. Biol. Med. v. 154, p. 496-499, 1977.

NADA, O.; TOYOHARA, T. An immunohistochemical study of serotonin-containing nerves in the colon of rats. 
Histochemistry, n. 86, p. 229-232, 1987.

OHMAN, L.; SIMRÉN, M. New insights into the pathogenesis and pathophysiology of irritable bowel syndrome. Digestive and Liver Disease, v. 39, n. 3, p. 201215, 2007.

PAN, H.; GERSHON, M. D. Activation of intrinsic afferent pathways in submucosal ganglia of the guinea pig small intestine. J. Neurosci. v. 20, p. 3295-3309, 2000.

PASSOS, M. C. F.; RAMOS, A. F. P. Patogenia dos distúrbios gastrointestinais funcionais. Gastroenterologia: da patogenia à prática clínica. 2006. p. 21-34.

RAYBOULD, H. E. Nutrient tasting and signaling mechanisms in the gut: I. Sensing of lipid by the intestinal mucosa. Am. J. Physiol. v. 277, p. G751-G755, 1999.

SANDERS-BUSH, E. S.; MAYER, S. E. Agonistas e antagonistas dos receptores da 5-hidroxitriptamina (serotonina). In: GOODMAN, A., GILMAN, P. As bases farmacológicas da terapêutica. 9. ed. Rio de Janeiro: Guanabara Koogan, 1996. p. 183.

SCHULIGOI, R. et al. Gastric acid-evoked c-fos messenger RNA expression in rat brainstem is signaled by capsaicinresistant vagal afferents. Gastroenterology, v. 115, p. 649660, 1998.

SCHWERTZ, I.; BRADESI, S.; MAYER, E. A. Current insights into pathophysiology of irritable bower syndrome. Curr. Gastroenterology Reports, v. 5, p. 331-336, 2003.

SENGUPTA, J. N.; GEBHART, G. F. Gastrointestinal afferent fibers and sensation. In: JOHNSON, L. (Ed.). Physiology of the Gastrointestinal tract. New York: Raven Press, 1994. p. 483-520.

SIDHU, M.; COOKE, H. J. Role for 5-HT and ACh in submucosal reflexes mediating colonic secretion. Am. J.

Physiol. Gastointest. Liver Physiol. v. 269, p. G346-G351, 1995.

SUGIUAR, T.; BIELEFELDT, K.; GEBHART, G. F. TRPV1 function in mouse colon sensory neurons is enhanced by metabotropic 5-hydroxytryptamine receptor activation. J. Neurosci. v. 24, p. 9521-9530, 2004.

SZURSZEWSKI, J. H.; MILLER, S. M. Physiology of prevertebral ganglia. In: JOHNSON, L. R. Physiology of the gastrointestinal tract. 3. ed. New York: Raven Press, 1994. p. 795-877.

TAMIR, H. et al. Human serotonectin: a blood glycoprotein that binds serotonin and is associated with platelets and white blood cells. J. Cell Sci. v. 73, p. 187-206, 1985.

TAKAHASHI, T.; OWYANG, C. Characterization of vagal pathways mediating gastric accomodation reflex in rats. Journal of Physiology, v. 504, p. 479-488, 1997.
THOMAS, D. P.; VANE, J. R. 5-hydroxytryptamine in the circulation of the dog. Nature, v. 216, p. 335-338, 1967.

VAN KUYK, E. M. et al. Defecation problems in children with Hirschsprung's disease: a biopsychosocial approach.

Pediatric Surgery International, New York, v. 16, n. 5, p. 312-316, 2000.

WALTHER, D. J. et al. Synthesis of serotonin by a second tryptophan hydroxylase isoform. Science, v. 299, p. 76, 2003.

WARDELL, C. F.; BORNSTEIN, J. C.; FURNESS, J. B. Projections of 5-hydroxytryptamine- immunoreactive neurons in guinea-pig distal colon. Cell Tissue Res. v. 278, p. 379-387, 1994.

WEISBRODT, N. W. Motility of the large intestine. In: JACOBSON, E.; JOHNSON, L. R.; WEISBRODT, N. W. Gastrointestinal physiology. St Louis: Mosby, 1997.

WINGREN, U. et al. Endoluminal secretin of serotonin and histamine into the small intestine of normal and nematodeinfected rats. Biogenic Amines, v. 5, p. 297-306, 1988.

WOOD, J. D.; ALPERS, D. H.; ANDREWS, P. L. R. Fundamentals of neurogastroenterology. Gut, v. 45, n. 2, p. II6-II16, 1999.

YANG, M. et al. Serotonin stimulates megakaryocytopoiesis via the 5-HT2 receptor. Blood Coagul Fibrinolysis, v. 7, p. 127-133, 1996.

ZHU, J. X. et al. Intestinal serotonin acts as a paracrine substance to mediate vagal signal transmission evoked by luminal factors in the rat. J. Physiol. v. 530, p. 431-442, 2001. 